\title{
COMPORTAMENTO MIGRATÓRIO DA LAGOSTA PANULIRUS ARGUS (LATREILLE), EM FRENTE AO EST ADO DO CEARÁ, BRASIL
}

\author{
ANTONIO ADAUTO FONTELES-FILHO \\ CARLOS TASSITO CORREA IVO ${ }^{1}$ \\ Laboratório de Ciências do Mar. Universidade Federal do Ceará \\ Fortaleza, Ceará, Brasil
}

\section{SYNOPSIS}

Tagging experiments have been used to study the migratory behaviour of the spiny lobster Panulirus argus (Latreille), off Ceará State, Brazil. An analysis based on vectorial theory has provided the calculation of the coefficient of directional dispersion ( V), the coefficient of randon disperson $\left(\mathrm{a}^{2}\right)$ and the centre of density, by quarters of the year. The lobsters were found to engage in breeding migration in the second quarter, when there is a low random dispersion and in feeding migration in the third and fourth quarters. when there is high randon dispersion. The spawning areas are located in offshore regions and the lobsters reach them by moving seawardly, from positions occupied nearer the cost, in the fourth quarter.

Introdução

Os experimentos de marcação constituen o método científico mais adequado para o estudo da migração de espécies animais, evi denciando o deslocamento de indivíduos a partir de um certo ponto de sua área de distribuição. As lagostas se movimentam dentro de uma área restrita à plataforma continental e seu ciclo migratório está relacionado com duas importantes funções do ciclo vital: alimentação (migração trófica) e reprodução (migração genética).

Os movimentos da lagosta Panulirus argus (Latreille) já foram anteriormente investigados, com base nos experimentos de marcação realizados pelo Laboratório de Ciências do Mar, em 1964 e 1965 (Paiva \&Fonteles Filho, 1968). Neste trabalho, aplicamos aos dados um tratamento matemático baseado na teoria vetorial, que nos permite determinar com maior clareza e segurança o comportamento migratório desta espécie, levando em consideração os parâmetros de dispersão e a posição do centro de densidade, em diferentes épocas do ano.

\section{Material}

Utilizamos os dados referentes às posições de recaptura, resultantes da marcação de 3.867 individuos, distribuídos da seguinte maneira: experimento $n^{O} 1-471$ indivíduos, sendo 230 machos e 241 fémeas, das quals 117 estavam ovadas $(48,5 \%)$, marcadas na posição $03^{\circ} 37^{\prime} \mathrm{S}-38^{\circ} 06^{\prime} \mathrm{W}$, no período de 16 a 25 de março de 1964 ; experimento n. 2 - 2.425 individuos, sendo 302 machos e 2.123 fêmeas das quais 1.961 estavam ovadas $(92,4 \%)$, marcados na posição $02^{0} 16^{\prime} \mathrm{S}-49^{\circ} 04 \mathrm{~W}$ ', no periodo de 14 a 23 de março de 1965; experimento n. 3 - 971 indivíduos, sendo 227 machos e 744 fêmeas, marcados na posição $02^{\circ} 35^{\prime} \mathrm{S}-40^{\circ} 10^{\prime} \mathrm{W}$, no período de 2 a 9 de julho de 1964

A marca e o método de marcação empregados nos experimentos se encontram descritos em Paiva \& Fonteles Filho (1968). Deve-se ressaltar que nem todas as marcas recuperadas foram devolvidas, ocorrendo também a perda de alguns indivíduos por morte devida ao processo de març̧ão.

\section{Metologia}

Para cada experimento, plotamos as posições de recaptura em um mapa da plataforma continentas em frente ao Estado do Ceará, por trimestres, sem levar em consideração o sexo do indivíduo, dado o pequeno número de machos em relação ao de fêmeas. Não utilizamos os dados referentes a indivíduos recapturados no intervalo de até 5 dias após sua liberação, para evitar vícios decorrentes da captưra em posiçōes muito próximas à de marcação.

Cada ponto de recaptura foi considerado como a extremidade de um vetor, caracterizado pelo ângulo de direcão do deslocamento $(\theta)$ e pela distância à posição de marcação $(r)$. Deste modo, cada vetor tem duas componentes determinadas em relação a um sistema de coordenadas cartesianas: $\mathrm{x}=\mathrm{r} \cos \theta$ (componente longitudinal) e $\mathrm{y} \equiv \mathrm{r} \operatorname{sen} \theta$ (componente latitudinal) em relação ao eixo dos $X(\mathrm{E}-\mathrm{W})$.

Segundo Jones (1965), o movimento de um indivíduo apresenta duas tendências: uma, direcional, medida pelo coeficiente de dispersão direcional ( V ) e outra, aleatória, medida pelo coeficiente de dispersão aleatória $\left(\mathrm{a}^{2}\right)$. Estes parâmetros são estimados através das seguintes equaçōes:

Bolsista do Conselho Nacional de Desenvolvimento Científico e Tecnológico ( $\mathrm{CNPq})$.

$$
\begin{aligned}
& \mathrm{V}=\frac{\sqrt{(\Sigma \mathrm{r} \cos \theta)^{2}+(\Sigma \mathrm{r} \operatorname{sen} \theta)^{2}}}{\Sigma \mathrm{t}} \\
& \mathrm{a}^{2}=\frac{\mathrm{i}}{\mathrm{n}}\left[\Sigma \frac{\mathrm{r}^{2}}{\mathrm{t}}-\frac{(\Sigma \mathrm{r} \cos \theta)^{2}+(\Sigma \mathrm{r} \operatorname{sen} \theta)^{2}}{\Sigma \mathrm{t}}\right]
\end{aligned}
$$

sendo, ainda, $t$ o tempo decorrido entre a marcação e a recaptura e $n$, o número de indivíduos recapturados.

$O$ valor de $V$ determina a velocidade de deslocamento numa certa direção, de modo que o centro de densidade dos indivíduos recapturados, num dado período de tempo, é determinado pelo ponto $V \bar{t}$ (sendo $\bar{t}$ o tempo médio decorrido entre as datas de marcação e recaptura) e pelo ângulo médio de dispersão $(\Psi)$, calculado pela equação:

$$
\Psi=\operatorname{arctg} \frac{\Sigma \mathrm{r} \operatorname{sen} \theta}{\Sigma \mathrm{r} \cos \theta}
$$

Sabendo-se que ( Beverton \& Holt, 1957 ),

$\mathrm{V}=$ nd $\quad$ e $\quad \mathrm{a}^{2}=\mathrm{Vd}=\mathrm{nd}^{2}$

(onde, $n$ é o número de movimentos por unidade de tempo e $d$, a distância média de deslocamento livre), estabelecemos a relação entre $a^{2}$ e $V$ como se segue:

$\mathrm{d}=\frac{\mathrm{a}^{2}}{\mathrm{~V}}$

\section{Resultados e Discussão}

As lagostas liberadas em grupo, numa pequena área que pode ser considerada como um ponto, passam a se movimentar independentes uma da outra, ocorrendo uma dispersão gradual dos indivíduos. Se não houvesse tendência direcional de dispersão, a resultante do deslocamento do grupo seria igual a zero, já que, para cada indivíduo que tivesse migrado $x$ milhas num certo sentido, se esperaria encontrar outro $x$ milhas em sentido exatamente oposto. Pela Tabela $\mathrm{I}$, vemos que a lagosta $P$. argus realizou dispersão direcional, con valores do coeficiente $V$ variando de 0,018 a $1,199 \mathrm{mi}$ lhas/dia.

As lagostas marcadas em março (experimentos n.o.s. 1 e 2), e liberadas em locais diferentes da plataforma continental, distantes cerca de 140 milhas, apresentaram comportamento migratório semelhante, pelos seguintes aspectos: (a) movimento paralelo à costa no segundo trimestre, caracterizado por baixa dispersāo aleatória, com o centro de densidade se aproximando da isobata de $50 \mathrm{me}-$ tros - com referência, principalmente, às lagostas marcadas na posição $02^{\circ} 16^{\prime} \mathrm{S}-40^{\circ} 04^{\prime} \mathrm{W}$, a maioria das quais estava em reprodução; (b) movimentos oblíquo à costa, no terceiro trimestre, e paralelo à costa, no quarto trimestre, caracterizados por elevada dispersão aleatória e com os centros de densidade mais próximos da costa, em torno da profundidade de 30 metros; (c) movimento perpendicular à costa no primeiro trimestre, no qual ocorre baixa dispersão aleatória e o centro de densidade se localiza bastante próximo da posição da marcação e a, apenas, 12 milhas daquele no primeiro trimestre (Tab. 1 ; Fig. 1 e 2). 
Tabela I-Estimativas trimestrais dos parâmetros de dispersâo da lagosta Panulirus argus (Latreille) - coeficiente de dispersão direcio$\mathrm{nal}(\mathrm{V})$, coeficiente de dispersáo aleatória $\left(\mathrm{a}^{2}\right)$, ângulo de dispersáo direcional $(\Psi)$, centro de densidade $(\mathrm{V} \overline{\mathrm{t}})$ e distândia média de dẹlocamento livre (d).

\begin{tabular}{|c|c|c|c|c|c|c|c|}
\hline \multirow[b]{2}{*}{ Trimestre } & \multirow[b]{2}{*}{$\begin{array}{c}\text { Número } \\
\text { de } \\
\text { recapturas }\end{array}$} & \multicolumn{6}{|c|}{ Parâmetros de dispersão } \\
\hline & & $\underset{\text { (milha/dia) }}{\mathrm{V}}$ & $\begin{array}{c}\mathrm{a}^{2} \\
\text { (milha }{ }^{2} / \text { dia) }\end{array}$ & $\begin{array}{c}\bar{t}^{\prime} \\
\text { (dia) }\end{array}$ & $\begin{array}{r}V \overline{\bar{t}} \\
\text { (milha) }\end{array}$ & $\underset{\text { (grau) }}{\Psi}$ & $\underset{\text { (milha) }}{\stackrel{\mathrm{d}}{{ }^{2}}}$ \\
\hline \multicolumn{8}{|c|}{ Experimento 1 - março/64 } \\
\hline $\begin{array}{l}2 \% \\
3 \% \\
4 ! \\
1 \%\end{array}$ & $\begin{array}{r}47 \\
9 \\
3 \\
3\end{array}$ & $\begin{array}{l}0,032 \\
0,042 \\
0,092 \\
0,102\end{array}$ & $\begin{array}{r}2,726 \\
14,061 \\
0,600 \\
1,868\end{array}$ & $\begin{array}{r}27 \\
214 \\
237 \\
225\end{array}$ & $\begin{array}{r}0,86 \\
9,14 \\
21,90 \\
25,92\end{array}$ & $\begin{array}{l}161 \\
141 \\
150 \\
136\end{array}$ & $\begin{array}{r}85,2 \\
344,8 \\
6,5 \\
18,3\end{array}$ \\
\hline Total & 62 & 0,057 & 4,227 & 74 & 4,22 & 145 & 74,2 \\
\hline \multicolumn{8}{|c|}{ Experimento 2 -março/65 } \\
\hline $\begin{array}{l}2 \% \\
3 \circ \\
40 \\
10\end{array}$ & $\begin{array}{r}92 \\
17 \\
3 \\
23\end{array}$ & $\begin{array}{l}0,123 \\
0,311 \\
0,049 \\
0,018\end{array}$ & $\begin{array}{r}1,979 \\
35,700 \\
1,914 \\
0,303\end{array}$ & $\begin{array}{l}155 \\
150 \\
359 \\
364\end{array}$ & $\begin{array}{r}19,13 \\
46,52 \\
17,63 \\
6,55\end{array}$ & $\begin{array}{l}343 \\
320 \\
196 \\
335\end{array}$ & $\begin{array}{r}16,1 \\
114,8 \\
39,1 \\
16,8\end{array}$ \\
\hline Total & 135 & 0,100 & 5,938 & 194 & 19,40 & 335 & 59,4 \\
\hline \multicolumn{8}{|c|}{ Experimento 3 - julho/64 } \\
\hline $\begin{array}{l}3 \% \\
4 ! \\
1 \% \\
2 !\end{array}$ & $\begin{array}{r}183 \\
16 \\
5 \\
13\end{array}$ & $\begin{array}{l}1,199 \\
0,066 \\
0,095 \\
0,063\end{array}$ & $\begin{array}{r}30,631 \\
7,614 \\
0,080 \\
0,491\end{array}$ & $\begin{array}{r}22 \\
162 \\
213 \\
307\end{array}$ & $\begin{array}{l}26,09 \\
10,68 \\
20,25 \\
19,34\end{array}$ & $\begin{array}{l}21 \\
11 \\
22 \\
65\end{array}$ & $\begin{array}{r}25,5 \\
115,4 \\
0,8 \\
7,8\end{array}$ \\
\hline Total & 217 & 0,448 & 26,424 & 54 & 24,19 & 23 & 59,0 \\
\hline
\end{tabular}

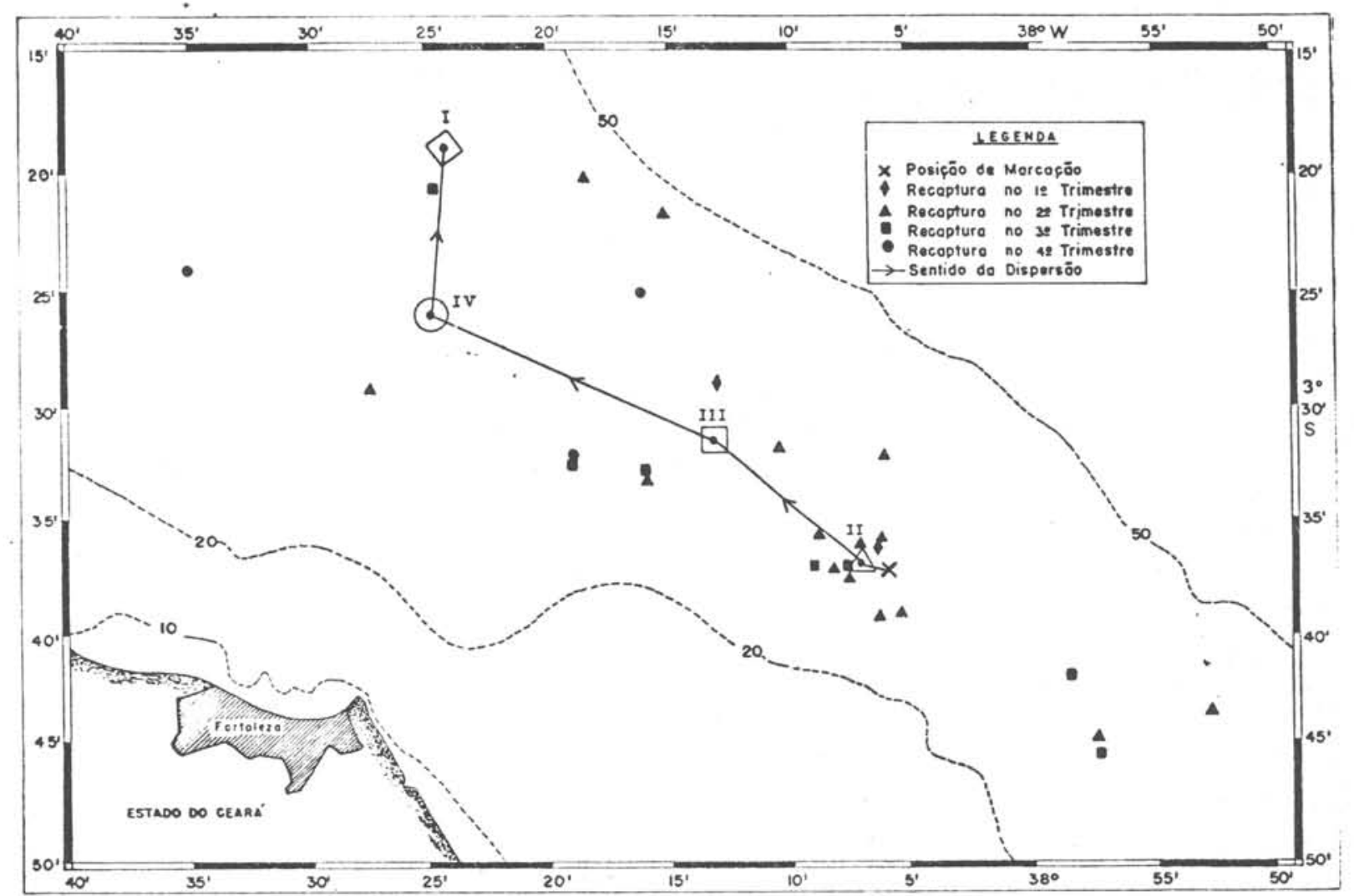

Fig. 1 - Distribuição espacial dos indivíduos da lagosta Panulirus argus (Latreille) recapturados no experimento n. 1 , por trimestres, com respectivos centros de densidade representados por símbolos maiores. Foi excluída do gráfico uma recaptura feita na posição $04^{\circ} 13^{\prime} \mathrm{S}-37^{\circ} 15^{\prime} \mathrm{W}\left(3^{\circ}\right.$. trimestre) 


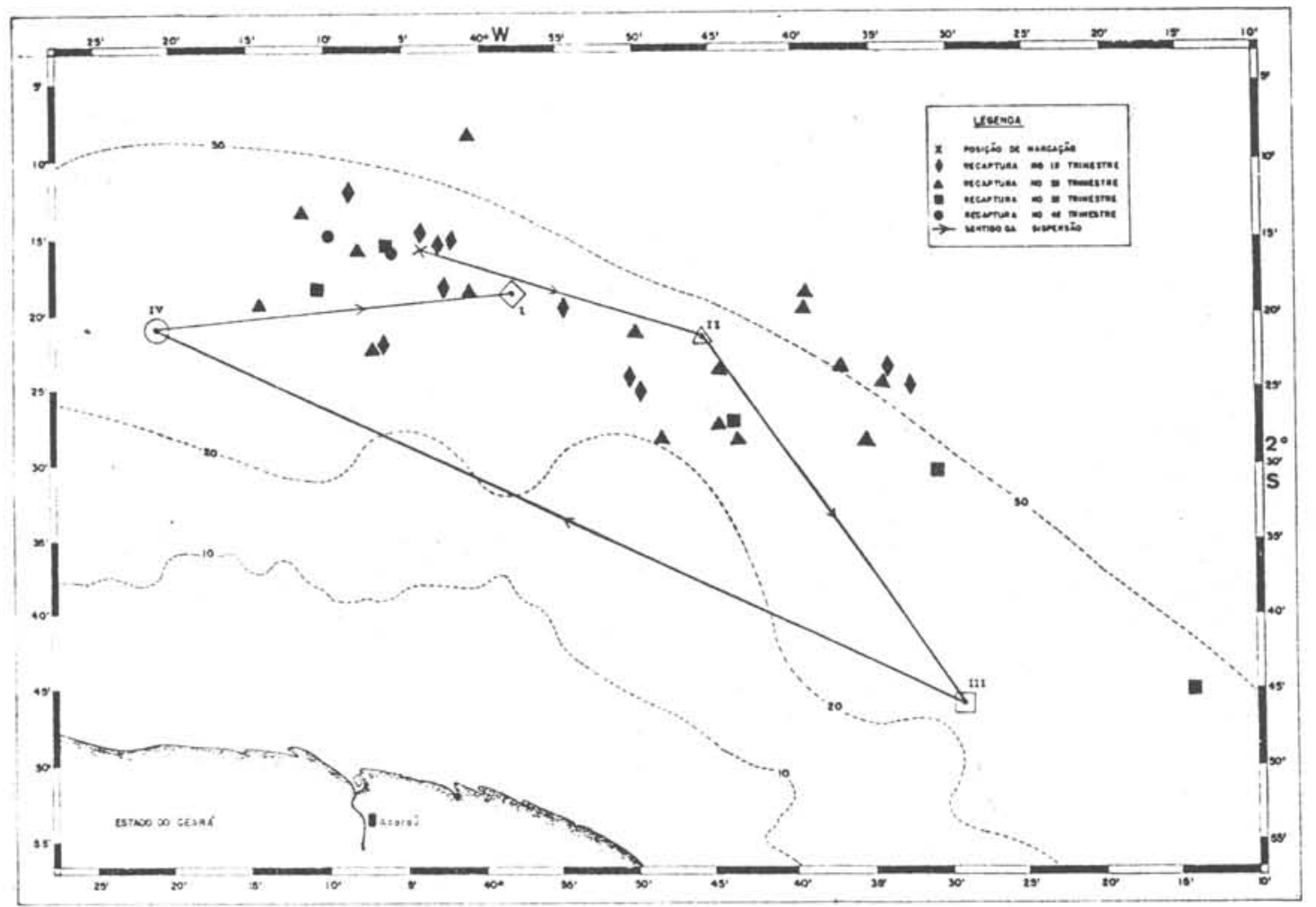

Fig. 2 -Distribuição espacial dos indivíduos da lagosta Panulirus argus (Latreille) recapturados no experimento n.2, por trimestres, com respectivos centras de densidade representados por símbolos maiores. Foram excluídas do gráfico as recapturas feitas nas posiçōes $02^{\circ} 29^{\prime} \mathrm{S}-39^{\circ} 01^{\prime} \mathrm{W}, 02^{\circ} 14^{\prime} \mathrm{S}-$ $40^{\circ} 40^{\prime} \mathrm{W}$ ( $22^{\circ}$ trimestre) e $02^{\circ} 30^{\prime} \mathrm{S}-40^{\circ} 49^{\prime} \mathrm{W}$ ( 4 ? $^{\circ}$ trimestre).

As lagostas marcadas em julho (experimento n. 2) apresentaram grande velocidade de dispersão direcional e grande tendência de dispersão aleatória, no terceuro trimestre, com o centro de densidade localizando-se em torno da profundidade de 30 metros; no quarto trimestre, o centro de densidade se moveu para uma posição mais próxima da de marcação e os indivíduos se moveram com pequena dispersão direcional e uma menor dispersão aleatória; no primeiro trimestre, ocorreu uma rápida migração de volta a uma area mais afastada da costa, próxima a ssobata de $50 \mathrm{~m}$, tornando-se o deslocamento paralelo à costa no sequndo trimestre, na direção $\mathrm{NE}$, com o centro de densidade mantendo-se na mesma faixa de profundidade e localizando-se a, apenas, 17 milhas de distância daquele no primeiro trimestre (Tab. I; Fig. 3).

Os valores de $V$ e $a^{2}$ em cada trimestre refletem o tipo de dispersão predominante, se a direcional se a aleatória. No entanto, a existência de dispersão direcional pode causar a superestimação

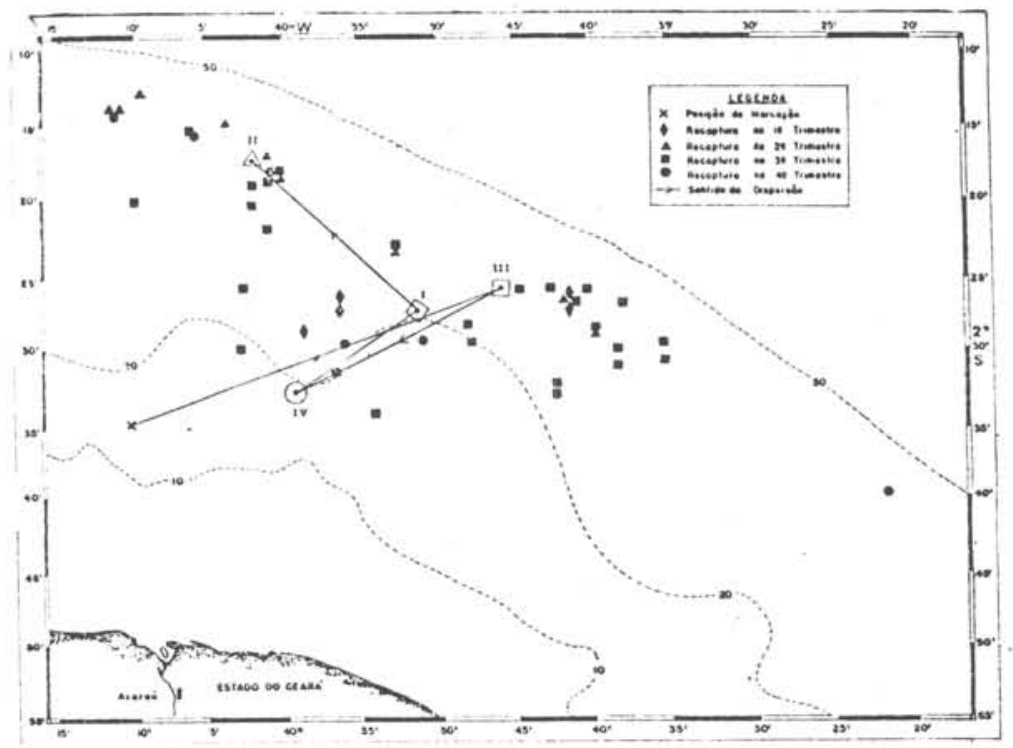

Fig. 3 - Distribuição espacial dos indivíduos da lagosta Panulirus argus (Latreille) recapturados no experimento n.3, por trimestres, com respectivos centros de densidade representados por símbolos maiores. Foram excluídas do gráfico as recapturas feitas nas posiçōes $03^{\circ} 32^{\prime} \mathrm{S}-38^{\circ} 24^{\prime} \mathrm{W}\left(2^{\circ}\right.$ trimestre) e $02^{\circ} 35^{\prime} \mathrm{S}-40^{\circ} 39^{\prime} \mathrm{W}\left(4 ?^{\circ}\right.$ trimestre). 
de $a^{5}$ (Jones, 1965), pnncipalmente logo em seguida à uberaçâo do indivíduo, quando é maior a velocidade de dispersão. Observamos este fato com relação ao terceiro trimestre, no experimento n. ${ }^{\circ} 3$, o que pode ter sido responsável por não ser bem definida a combinação de altos valores de $V$ com baixos valores de $a^{2}$, e vice-versa. Os valores de $d$, porém, indicam a predominância da componente aleatória no terceiro trimestre, ocorrendo ó inverso no primeiro e segundo trimestres ( $\mathrm{T} a \mathrm{~b}, \mathrm{I}$ ).

A desova da lagosta $P$. argus se concentra no período de março a junho (Mesquita, 1973), mas não existem informações sobre áreas e épocas de alimentação. No entanto, as maiores freqüências de indivíduos em mudà ocorrem nos meses de janeiro e julho-agosto, sendo possível concluir que o terceiro e quarto trimestres, sendo períodos de crescimento mais rápido, podem ser considerados como época de alimentação (principalmente o terceiro, por vir logo em seguida à época de desova).

Ficou bem evidente, portanto, que: (1) as lagostas realizam migração genética durante o segundo trimestre, para as áreas de desova que se localizam em regiōes afastadas da costa, em profundi dades entre $4 n$ a 50 metros. através de migração direcional em que predomina a baixa dispersão aleatoria; (2) as lagostas se arspersam $\mathrm{em}$ sentido paralelo à costa no terceiro e quarto trimestres, quando realizam migração trotica, em que predomina uma elevada dispersuo aleatória, relacionada com o movimento de indivíduos à procura de alimento com distribuição espacial não-uniforme; (3) no primeiro trimestre, as lagostas diminuiriam seu ritmo alimentar e a dispersão assume uma tendência direcional predominante, perpendicular à costa, em direção às áreas de desova.

\section{Bibliografia}

BEVERTON, R. H. H. \& HOLT, S. J. 1957. On the dunomics of exploited fish populations. Fishery Invest., Lond., ser. 2, 19: $1-533,155$ figs.

JONES, R. 1965. A method of analysis of some tagged haddock returns. J. Cons. perm. int. Explor. Mer, 25 (1):58-72, 6 figs.

MESQUITA, A. L. L. 1973. Aspectos cronológicos da reprodução da lagosta Panulinus argus (Latreille), no Estado do Ceará (Brasil). Arq. Ciênc. Mar., 13 (2): 77-82, 3 figs.

PAIVA, M. P. \& FONTELES FILHO, A. A. 1968. Sôbre as migrações e índices de exploração da lagosta Panulirus argus (Latreille), ao longo da costa do Estado do Ceará. Arq. Estaç. Biol. mar. Univ. Fed. Ceará, 8(1):15-23, 6 figs. 